\title{
A descent extension of the Dai - Yuan conjugate gradient technique
}

\author{
Basim A. Hassan ${ }^{1}$, Zeyad M. Abdullah², Hawraz N. Jabbar ${ }^{3}$ \\ ${ }^{1}$ Department of Mathematics, College of Computers Sciences and Mathematics, University of Mosul, Iraq \\ ${ }^{2}$ Faculty of Computers Sciences and Mathematics, University of Tikrit, Iraq \\ ${ }^{3}$ Department of Mathematics, College of Sciences, University of Kirkuk, Iraq
}

\begin{tabular}{l} 
Article Info \\
\hline Article history: \\
Received Jan 21, 2019 \\
Revised May 1, 2019 \\
Accepted May 22, 2019 \\
\hline
\end{tabular}

Keywords:

Conjugate coefficient

Conjugate gradient method

Global convergence property

\begin{abstract}
The conjugate coefficient is the very establishment of an variety of the conjugate gradient methods for solving unconstrained optimization problems. Based on Dai-Yuan technique, a new extension method was proposed. In contrary to the CG classical method, this proposed method employs a coefficient that has an important role in constructing the new method with less computational efforts. This proposed method was found to be efficient on the basis of theory analysis and also numerical results to show the efficiency of the proposed method.
\end{abstract}

Copyright $(2019$ Institute of Advanced Engineering and Science. All rights reserved.

\section{Corresponding Author:}

Basim A. Hassan,

Department of Mathematics,

College of Computers Sciences and Mathematics,

University of Mosul, Iraq.

Email: basimabas39@gmail.com

\section{INTRODUCTION} formula:

The technique of conjugate gradient is useful in finding the problem's minimum value. Consider this

$$
\operatorname{Min} f(x), \mathrm{x} \in \mathrm{R}^{\mathrm{n}}
$$

where $f: R^{n} \rightarrow R$ is smooth [1]. The iteration technique is often used to solve Equation (1) and it is written as:

$$
x_{k+1}=x_{k}+\alpha_{k} d_{k}
$$

where $\alpha_{k}$ is the step length. In any complete conjugate gradient method, the step length $\alpha_{k}$ is regularly chosen to satisfy the firm line search conditions. As the most eminent line search conditions, Wolfe line search necessitates that:

$$
f\left(x_{k}+\alpha_{k} d_{k}\right) \leq f\left(x_{k}\right)+\delta \alpha_{k} g_{k}^{T} d_{k}
$$




$$
d_{k}^{T} g\left(x_{k}+\alpha_{k} d_{k}\right) \geq \sigma d_{k}^{T} g_{k}
$$

where $0<\delta<\sigma$. More details are found in [2]. Therefore, this study will focus on the CG method whose direction of search is written as:

$$
d_{k+1}=-g_{k+1}+\beta_{k} d_{k}
$$

$\beta_{k}$ is a scalar known as the CG update and $g_{k+1}$ is gradient. Different CG techniques chiefly convert into varied selections for the CG update, as reviewed in [3].

In convergence properties, the conjugate gradient method proposed by Dai and Yuan [4] is generally believed to be one of the best CG techniques. The CG update of the Dai and Yuan (DY) technique is given by:

$$
\beta_{k}^{D Y}=\frac{g_{k+1}^{T} g_{k+1}}{d_{k}^{T} y_{k}}
$$

where $y_{k}=g_{k+1}-g_{k}$.

We utter that the search directions $d_{k+1}$ satisfy the sufficient descent condition if:

$$
g_{k+1}^{T} d_{k+1} \leq-c\left\|g_{k+1}\right\|^{2}
$$

where $c$ is a positive constant. More details on performance profile are given in [5]. Now we make extension of the Dai - Yuan conjugate gradient technique and analyze its convergence.

\section{EXTENSION OF THE DAI - YUAN CONJUGATE GRADIENT TECHNIQUE}

Attempting to make an extension on the CG update suggested by Dai and Yuan through multiplying (5) with $g_{k+1}$ and using (6), this equation is obtained:

$$
g_{k+1}^{T} d_{k+1}=\frac{g_{k+1}^{T} g_{k+1}}{d_{k}^{T} y_{k}} g_{k}^{T} d_{k}
$$

which with (6) gives an equivalent formula to (6):

$$
\beta_{k}=\frac{g_{k+1}^{T} d_{k+1}}{g_{k}^{T} d_{k}}
$$

For more details can be found in [6]. Obviously, if the Hessian matrix of $f$ is non negative definite, the majority efficient search direction at $x_{k}$ will be the Newton direction:

$$
d_{k+1}=-\nabla^{2} f\left(x_{k+1}\right)^{-1} g_{k+1}
$$

The Hessian matrices satisfy the secant equation:

$$
\nabla^{2} f\left(x_{k+1}\right) s_{k}=y_{k}
$$

where $s_{k}=x_{k+1}-x_{k}$. 
Suppose that $I$ denotes the identical matrix and assume that a matrix $\nabla^{2} f\left(x_{k+1}\right)$ is desired with a simplified construction satisfying the secant equation. Specifically, this formula is desired:

After some algebraic manipulations one obtains :

$$
\nabla^{2} f\left(x_{k+1}\right)=\eta_{k+1} I_{k+1}
$$

So, the equation of secant is formed as follows:

$$
\eta_{k+1} s_{k}=y_{k}
$$

This equation describes the most popular spectral gradient method for optimization with the direction of search:

$$
d_{k+1}=-\frac{1}{\eta_{k+1}} g_{k+1}
$$

where $d_{k+1}$, which is defined in (14), would be replaced by $d_{k+1}$ in (8), the following equation is obtained:

$$
\beta_{k}=-\frac{1}{\eta_{k+1}} \frac{g_{k+1}^{T} g_{k+1}}{g_{k}^{T} d_{k}}
$$

If a step - length $\alpha_{k}$ is inexact, then:

$$
\beta_{k}=\frac{1}{\eta_{k+1}} \frac{g_{k+1}^{T} g_{k+1}}{y_{k}^{T} d_{k}}
$$

Denoted this formula procedure as $\beta_{k}^{\mathrm{BDY}}$. Definitely, more sufficient test would be required $\eta_{k+1}$. The coefficient $\eta_{k+1}$ plays an important role in the current method. It can be computed by the formula:

$$
1 / \eta_{k+1}=2\left[f_{k}-f_{k+1}+g_{k+1}^{T} s_{k}\right] / y_{k}^{T} s_{k}
$$

This coefficient defines the most popular Yuan method [7]. The Extension algorithm is presented below and called Algorithm BDY.

\section{Algorithm BDY}

0 : Given constants $\varepsilon>0, \delta \in(0,1), \sigma \in(\delta, 1)$ and $x_{0} \in R^{n}$. Let $d_{0}=-g_{0}, k=0$.

1 : Examine a stopping criterion. If yes, then stop; if not, continue with the next step.

2 : Define a step length $\alpha_{k}$ using a proper line search.

3 : Let $x_{k+1}=x_{k}+\alpha_{k} d_{k}$, and compute $\beta_{k}^{B D Y}$ by (16).

4 : Compute the search direction $d_{k+1}=-g_{k+1}+\beta_{k} d_{k}$.

5 : Set $k=k+1$ and continue with step 1 .

\section{CONVERGENCE ANALYSIS}

In this paper, $f(x)$ is assumed as that:

1. For a given $x_{0} \in R^{n}$, the function $f(x)$ has lower bound on $\Psi=\left\{x \in R^{n}: f(x) \leq f\left(x_{0}\right)\right\}$.

2. A positive constant L exists; so: 


$$
\|g(x)-g(y)\| \leq L\|x-y\|, \forall x, y \in \Phi
$$

where $\Phi \in \Psi$ is an open convex set. That is to say, the gradient $g(x)$ is Lipschitz continuous in $\Phi$. More details are found in $[8,9]$.

\section{Theorem 1} holds, i.e.,

Suppose that the search direction $d_{k+1}$ is created by (5), and $\eta_{k+1} \leq 1$. Then the descent condition

$$
g_{k+1}^{T} d_{k+1} \leq 0
$$

\section{Proof:}

This theorem is proved by induction. As $d_{0}=-g_{0}$, there is $g_{0}^{T} d_{0}=-\left\|g_{0}\right\|^{2}<0$. Assume that $g_{k}^{T} d_{k}<0$ for all $k \in n$. Through multiplying (5) by $g_{k+1}$, the following equation is obtained:

$$
\begin{aligned}
g_{k+1}^{T} d_{k+1} & =-\left\|g_{k+1}\right\|^{2}+\eta_{k+1} \frac{\left\|g_{k+1}\right\|^{2}}{y_{k}^{T} d_{k}} g_{k+1}^{T} d_{k} \\
& =\left\|g_{k+1}\right\|^{2}\left[-1+\eta_{k+1} \frac{g_{k+1}^{T} d_{k}}{y_{k}^{T} d_{k}}\right] \\
& =\left\|g_{k+1}\right\|^{2}\left[\frac{\eta_{k+1} g_{k+1}^{T} d_{k}-y_{k}^{T} d_{k}}{y_{k}^{T} d_{k}}\right]
\end{aligned}
$$

Since $y_{k}^{T} d_{k}=g_{k+1}^{T} d_{k}-g_{k}^{T} d_{k}$, then :

$$
\begin{aligned}
g_{k+1}^{T} d_{k+1} & =\left\|g_{k+1}\right\|^{2}\left[\frac{\eta_{k+1} g_{k+1}^{T} d_{k}-g_{k+1}^{T} d_{k}+g_{k}^{T} d_{k}}{y_{k}^{T} d_{k}}\right] \\
& =\left\|g_{k+1}\right\|^{2}\left[\frac{\left(\eta_{k+1}-1\right) g_{k+1}^{T} d_{k}+g_{k}^{T} d_{k}}{y_{k}^{T} d_{k}}\right] \\
& =\frac{\left\|g_{k+1}\right\|^{2}}{y_{k}^{T} d_{k}}\left[\left(\eta_{k+1}-1\right) g_{k+1}^{T} d_{k}+g_{k}^{T} d_{k}\right]
\end{aligned}
$$

From above equation we get:

$$
\frac{\left\|g_{k+1}\right\|^{2}}{y_{k}^{T} d_{k}}=\frac{g_{k+1}^{T} d_{k+1}}{\left[\left(\eta_{k+1}-1\right) g_{k+1}^{T} d_{k}+g_{k}^{T} d_{k}\right]}=\beta_{k}^{D Y}
$$

Now,

$$
\begin{aligned}
\beta_{k}^{\mathrm{BDY}} & =\eta_{k+1} \frac{\left\|g_{k+1}\right\|^{2}}{y_{k}^{T} d_{k}}=\eta_{k+1} \beta_{k}^{D Y} \\
& \left.=\frac{\eta_{k+1} g_{k+1}^{T} d_{k+1}}{\left(\eta_{k+1}-1\right) g_{k+1}^{T} d_{k}+g_{k}^{T} d_{k}}\right]
\end{aligned}
$$


From above equation we get :

$$
\beta_{k}^{\mathrm{BDY}}=\omega_{k+1} \frac{g_{k+1}^{T} d_{k+1}}{g_{k}^{T} d_{k}}
$$

where $\omega_{k+1}=\eta_{k+1} /\left(1+\left(\eta_{k+1}-1\right) l_{k}\right)$ and $l_{k}=g_{k+1}^{T} d_{k} / g_{k}^{T} d_{k}$. Assume that $g_{k}^{T} d_{k}<0$. From $l_{k}$ and Wolfe state $g_{k+1}^{T} d_{k} \geq \sigma g_{k}^{T} d_{k}$ we get :

$$
l_{k} \leq \sigma \quad \rightarrow \quad l_{k} \leq \sigma<1
$$

and

$$
\omega_{k+1} \leq \eta_{k+1} /\left(1+\left(\eta_{k+1}-1\right) \sigma\right)
$$

where $\eta_{k+1} /\left(1+\left(\eta_{k+1}-1\right) \sigma>0\right.$. The above equation to get :

$$
g_{k+1}^{T} d_{k+1}=\frac{1}{\omega_{k+1}} \beta_{k}^{\mathrm{BDY}} g_{k}^{T} d_{k} \leq 0
$$

Therefore, the proof is complete.

Due to playing an important role in analyzing the convergence property for conjugate gradient, Zoutendijk's condition [10] will be proved to be a part of the proposed Algorithm in this study.

\section{Lemma 1}

Let that $d_{k+1}$ is generated by (5) and step size $\alpha_{k}$ fulfills (3) and (4), if $f(x)$ satisfies the Assumptions, then :

$$
\sum_{k=1}^{\infty} \frac{\left(g_{k+1}^{T} d_{k+1}\right)^{2}}{\left\|d_{k+1}\right\|^{2}}<\infty
$$

holds.

\section{Theorem 2}

Let that Assumptions 1 and 2 are held, and that the search direction $d_{k+1}$ is calculated by the formulation in the second step, then:

$$
\lim _{k \rightarrow \infty} \inf \left\|g_{k+1}\right\|=0
$$

\section{Proof:}

Now, equation (29) can be proved by contradiction, assuming that there is a constant $\gamma>0$ such that :

$$
\left\|g_{k+1}\right\| \geq \gamma, \forall k \geq 0
$$

Since $d_{k+1}=-g_{k+1}+\beta_{k} d_{k}$, then the result is the following equation:

$$
\left\|d_{k+1}\right\|^{2}=\left(\beta_{k}\right)^{2}\left\|d_{k}\right\|^{2}-2 d_{k+1}^{T} g_{k+1}-\left\|g_{k+1}\right\|^{2}
$$


Dividing both sides of (31) by $\left(d_{k+1}^{T} g_{k+1}\right)^{2}$ and using (24), the following formula is obtained:

$$
\begin{aligned}
\frac{\left\|d_{k+1}\right\|^{2}}{\left(d_{k+1}^{T} g_{k+1}\right)^{2}} & \leq \omega_{k+1} \frac{\left\|d_{k}\right\|^{2}}{\left(d_{k}^{T} g_{k}\right)^{2}}-\frac{2}{\left(d_{k+1}^{T} g_{k+1}\right)}-\frac{\left\|g_{k+1}\right\|^{2}}{\left(d_{k+1}^{T} g_{k+1}\right)^{2}} \\
& \leq \omega_{k+1} \frac{\left\|d_{k}\right\|^{2}}{\left(d_{k}^{T} g_{k}\right)^{2}}-\left(\frac{\left\|g_{k+1}\right\|}{d_{k+1}^{T} g_{k+1}}+\frac{1}{\left\|g_{k+1}\right\|}\right)^{2}+\frac{1}{\left\|g_{k+1}\right\|^{2}} \\
& \leq \omega_{k+1} \frac{\left\|d_{k}\right\|^{2}}{\left(d_{k}^{T} g_{k}\right)^{2}}+\frac{1}{\left\|g_{k+1}\right\|^{2}}
\end{aligned}
$$

which, along with (26), can yield:

$$
\left|\omega_{k+1}\right| \leq 1
$$

Using (32) and (33), the following equation can be obtained:

$$
\frac{\left\|d_{k+1}\right\|^{2}}{\left(d_{k+1}^{T} g_{k+1}\right)^{2}} \leq \frac{\left\|d_{k}\right\|^{2}}{\left(d_{k}^{T} g_{k}\right)^{2}}+\frac{1}{\left\|g_{k+1}\right\|^{2}}
$$

Using (34) recursively and noting that $\left\|d_{1}\right\|^{2}=-d_{1}^{T} g_{1}=\left\|g_{1}\right\|^{2}$,

$$
\frac{\left\|d_{k+1}\right\|^{2}}{\left(d_{k+1}^{T} g_{k+1}\right)^{2}} \leq \sum_{i=1}^{k+1} \frac{1}{\left\|g_{i+1}\right\|^{2}} \leq \frac{k}{\gamma}
$$

Then, from this equation and (30), the following formula can be derived:

$$
\frac{\left(d_{k+1}^{T} g_{k+1}\right)^{2}}{\left\|d_{k+1}\right\|^{2}} \geq \frac{\gamma}{k},
$$

and this denotes that:

$$
\sum_{k=1}^{\infty} \frac{\left(g_{k+1}^{T} d_{k+1}\right)^{2}}{\left\|d_{k+1}\right\|^{2}}=\infty
$$

This is in contrary to the Zoutendijk condition (28). Thus, (29) holds.

\section{NUMERICAL RESULTS AND DISCUSSION}

In this paper, the algorithm of extension was tested and compared to the method of Dai-Yuan [4]. For comparing methods employed in this study with other classical ones, the gradient errors will be checked for measuring the criteria of stop for algorithms. In general, the iteration will be forced to stop when the gradient norm satisfies this inequality:

$$
\left\|g_{k+1}\right\|<10^{-6}
$$


By employing the process of Wolfe line search, the parameters will be selected as follows:

$$
\delta_{1}=0.001 \text { and } \delta_{2}=0.9
$$

In this study, experiments were conducted on 30 problems of unconstrained optimization test of Andrei's collection with the dimensions 100 and 1000; for details see Andrei [11]. Optimization problems is an important tool in all papers optimization [12-15]. The comparing data contain number of iterations (IN), the number of restart (NR) and the number of function evaluations (NF) (Table 1). Using Fortran 90 to code this methods.

Table 1. Comparing different conjugate gradient methods with different test functions

\begin{tabular}{|c|c|c|c|c|c|c|c|}
\hline \multirow{2}{*}{ P. No. } & \multirow{2}{*}{$\mathrm{n}$} & \multicolumn{3}{|c|}{ DY algorithm } & \multicolumn{3}{|c|}{ BDY algorithm } \\
\hline & & $\mathrm{NI}$ & NR & $\mathrm{NF}$ & NI & NR & $\mathrm{NF}$ \\
\hline \multirow[t]{2}{*}{1} & 100 & 27 & 8 & 14 & 26 & 7 & 13 \\
\hline & 1000 & 26 & 8 & 13 & 25 & 7 & 13 \\
\hline \multirow[t]{2}{*}{2} & 100 & 28 & 7 & 11 & 25 & 5 & 9 \\
\hline & 1000 & 49 & 15 & 23 & 48 & 14 & 22 \\
\hline \multirow[t]{2}{*}{3} & 100 & 125 & 29 & 83 & 125 & 29 & 83 \\
\hline & 1000 & 629 & 106 & 393 & 550 & 90 & 342 \\
\hline \multirow[t]{2}{*}{4} & 100 & 21 & 5 & 10 & 13 & 3 & 6 \\
\hline & 1000 & 29 & 7 & 15 & 26 & 7 & 13 \\
\hline \multirow[t]{2}{*}{5} & 100 & 61 & 15 & 40 & 61 & 11 & 37 \\
\hline & 1000 & 101 & 27 & 64 & 79 & 22 & 49 \\
\hline \multirow[t]{2}{*}{6} & 100 & 19 & 6 & 10 & 19 & 6 & 10 \\
\hline & 1000 & 35 & 12 & 22 & 19 & 6 & 10 \\
\hline \multirow[t]{2}{*}{7} & 100 & 151 & 26 & 79 & 137 & 24 & 73 \\
\hline & 1000 & 156 & 28 & 85 & 153 & 25 & 81 \\
\hline \multirow[t]{2}{*}{8} & 100 & 28 & 8 & 10 & 28 & 8 & 11 \\
\hline & 1000 & 33 & 10 & 15 & 29 & 11 & 13 \\
\hline \multirow[t]{2}{*}{9} & 100 & 59 & 12 & 31 & 47 & 10 & 25 \\
\hline & 1000 & 51 & 10 & 26 & 54 & 11 & 28 \\
\hline \multirow[t]{2}{*}{10} & 100 & 173 & 37 & 111 & 163 & 33 & 105 \\
\hline & 1000 & 767 & 160 & 493 & 583 & 117 & 373 \\
\hline \multirow[t]{2}{*}{11} & 100 & 133 & 26 & 85 & 126 & 25 & 83 \\
\hline & 1000 & 381 & 71 & 243 & 347 & 69 & 229 \\
\hline \multirow[t]{2}{*}{12} & 100 & 136 & 24 & 87 & 136 & 24 & 87 \\
\hline & 1000 & 421 & 58 & 250 & 400 & 63 & 241 \\
\hline \multirow[t]{2}{*}{13} & 100 & 47 & 11 & 30 & 53 & 4 & 29 \\
\hline & 1000 & 62 & 15 & 37 & 62 & 14 & 36 \\
\hline \multirow[t]{2}{*}{14} & 100 & 33 & 9 & 17 & 40 & 11 & 20 \\
\hline & 1000 & 48 & 12 & 22 & 35 & 9 & 16 \\
\hline \multirow[t]{2}{*}{15} & 100 & 60 & 11 & 35 & 56 & 12 & 36 \\
\hline & 1000 & 55 & 14 & 35 & 54 & 8 & 31 \\
\hline Total & & 3944 & 787 & 2389 & 3519 & 684 & 2077 \\
\hline
\end{tabular}

Problems numbers indicant for : " 1 . is the Extended Beale, 2. is the Penalty, 3. is the Perturbed Quadratic, 4. is the Extended Tridiagonal 1, 5. is the Generalized Tridiagonal 2, 6. is the Extended Himmelblau, 7. is the Extended Powell, 8. is Extended Cliff, 9. is the Extended Wood, 10. is the Quadratic QF2, 11. is the DIXMAANE (CUTE), 12. is the Partial Perturbed Quadratic, 13. is the Broyden Tridiagonal, 14. is the LIARWHD (CUTE), 15. is the Generalized quartic GQ2.”.

The summary of our results in Table 2. The result presented in Table 2 imply that BDY method improved over the performance of DY method. The improvement of method BDY over DY is $13 \%$, in average, of the iterations number, and $13 \%$, in average, of the restart number and $10 \%$, in average, of function evaluations number.

Table 2. Ratio of algorithm BDY cost to DY cost

\begin{tabular}{cccc}
\hline & NI & NR & NF \\
\hline DY algorithm & $100 \%$ & $100 \%$ & $100 \%$ \\
BDY algorithm & $86.94 \%$ & $86.91 \%$ & $89.22 \%$ \\
\hline
\end{tabular}




\section{CONCLUSION}

An extended method of nonlinear DY conjugate gradient was proposed in this paper. The extended direction refers to the descent direction. Analysis of convergence analysis was carried out and the statistics showed that the proposed technique is efficient for the given problems of nonlinear optimization test.

\section{REFERENCES}

[1] Ahmad A. and Zabidin S., "Modification of Nonlinear Conjugate Gradient Method with Weak Wolfe-Powell Line Search," Hindawi Abstract and Applied Analysis, pp. 1-6, 2017.

[2] Nocedal J. and Yuan Y., "Analysis of a self-scaling quasi-Newton method," J. Mathematical programming, vol. 61, pp. 19-37, 1993.

[3] Hager W. W. and Zhang H., "A survey of nonlinear conjugate gradient methods," Pac. J. Optim., vol. 2, pp. 35-58, 2006.

[4] Y. H. Dai and Yuan Y., "A Nonlinear Conjugate Gradient with a Strong Global Convergence Property,” SIAM Journal of Optimization, vol. 10, pp. 177-182, 2000.

[5] Babaie S. K., "An eigen value study on the sufficient descent property of a modified Polak-Ribi- Polak conjugate gradient method," Bull. Iranian Math. Soc., vol. 40, pp. 235-242, 2014.

[6] Y. H. Dai and Yuan Y., "An Efficient Hybrid Conjugate Gradient Method for Unconstrained Optimization," Annals of Operations Research, vol. 103, pp. 33-47, 2001.

[7] Y. Yuan, "A modified BFGS algorithm for unconstrained optimization," IMA Journal Numerical Analysis, vol. 11, pp. 325-332, 1991.

[8] Guangming Z., "A descent algorithm without line search for unconstrained optimization," Applied Mathematics and Computation, vol. 215, pp. 2528-2533, 2009.

[9] Basim A. H., "A new formula for conjugate parameter computation based on the quadratic model," Indonesian Journal of Electrical Engineering and Computer Science, vol. 3, pp. 954-961, 2019.

[10] Zoutendijk G., "Nonlinear programming computational methods," Integer and Nonlinear Programming, J. Abadie, ed, North-Holland, Amsterdam, pp. 37-86, 1970.

[11] Andrie N., "An Unconstrained Optimization Test functions collection," Advanced Modeling and optimization, vol. 10, pp. 147-161, 2008.

[12] Li C., "A modified conjugate gradent method for unconstrained optimization," TELKOMNIKA Telecommunication, Computing, Electronics and Control", vol. 11, pp. 6373-6380, 2013.

[13] Basim A. H. and Hussein K. K., "A new class of BFGS updating formula based on the new quasi-newton equation," Indonesian Journal of Electrical Engineering and Computer Science, vol. 3, pp. 945-953, 2019.

[14] Ghani N., Kamaruddin S., Ramli M., Musirin I. And Hashim H. "Enhanced BFGS quasi-Newton backpropagation models on MCCI data". Indonesian Journal of Electrical Engineering and Computer Science. 2017;8:101-106.

[15] Li C. Fang L. and Cao X. "Global convergenceof a kind of conjugate gradient method". TELKOMNIKA Telecommunication, Computing, Electronics and Control. 2013; 11: 544-549,2013. 